\title{
THE EXISTENCE OF LOCALLY FINE SIMPLICIAL SUBDIVISIONS
}

\author{
W. F. PFEFFER
}

(Received 2 July 1981)

Communicated by E. Strzelecki

\begin{abstract}
Given a simplex $S$ and a positive function $\delta$ on $S$, we show that there is a simplicial subdivision of $S$ such that the diameter of each subdividing simplex is smaller that $\delta$ evaluated at some of its vertices. 1980 Mathematics subject classification (Amer. Math. Soc.): primary 26 A 39, 28 A 15; secondary 57 C 05 .
\end{abstract}

Keywords and phrases: complex, partition, superadditive function, lower derivate.

\section{Introduction}

The purpose of this note is to prove a theorem which we believe is fundamental to the geometric theory of the conditionally convergent integral defined by Kurzweil (1957) and Henstock (1968).

The very beginning of a geometric integration theory is, of course, a workable higher dimensional integral. While the Henstock-Kurzweil definition extends trivially to intervals of any dimension, the integration over intervals is not adequate. It has been well established that the geometry of higher dimensional spaces is related to simplexes and their simplicial subdivisions, rather than to intervals and their usual subdivisions. The reason why intervals work in the real line is that one-dimensional intervals are simplexes, and their usual subdivisions are simplicial.

(C) Copyright Australian Mathematical Society 1982 
To define the Henstock-Kurzweil integral over simplexes, we must establish first the existence of simplicial partitions compatible with an arbitrary a priori given positive function. As we are concerned with simplicial partitions only, the standard argument of Henstock (1968, Theorem 1) does not work. Instead a completely different and surprisingly intricate technique has to be employed.

The geometric nature of our investigation brings in many notions which are common in algebraic or piecewise linear topology but usually alien in analysis. In order to keep the exposition reasonably self-contained, we shall include several definitions which would have been superfluous if this paper were aimed at the audience of topologists alone.

\section{Preliminaries}

By $\mathbf{R}$ and $\mathbf{R}_{+}$we denote, respectively, the set of all real and all positive real numbers. Throughout, $m \geqslant 1$ will be a fixed integer, and $\mathbf{R}^{m}$ will denote the $m$-dimensional Euclidean space. For a point $x=\left(\xi_{1}, \ldots, \xi_{m}\right)$ in $\mathbf{R}^{m}$, we let

$$
|x|=\left(\sum_{i=1}^{m} \xi_{i}^{2}\right)^{1 / 2}
$$

If $A \subset \mathbf{R}^{m}$, then $A^{-}$and $d(A)$ denote the closure and the diameter of $A$, respectively. If $B \subset A \subset \mathbf{R}^{m}$, then

$$
\text { int }_{A} B=A-(A-B)^{-}
$$

is the interior of $B$ relative to $A$. Given a family $\mathscr{Q}$ of subsets of $\mathbf{R}^{m}$, we let

$$
\operatorname{mesh} \mathcal{Q}=\sup \{d(A): A \in \mathbb{Q}\} .
$$

For $x_{0}, \ldots, x_{k}$ in $\mathbf{R}^{m},-1 \leqslant k \leqslant m$, set

$$
\left\langle x_{0} \cdots x_{k}\right\rangle=\left\{\sum_{i=0}^{k} c_{i} x_{i} \in \mathbf{R}^{m}: \sum_{i=0}^{k} c_{i}=1, c_{i} \geqslant 0, i=0, \ldots, k\right\} .
$$

If the vectors $x_{i}-x_{0}, i=1, \ldots, k$, are linearly independent, we call $\left\langle x_{0} \cdots x_{k}\right\rangle$ a $k$-simplex. Each $l$-simplex $\left\langle x_{i_{0}} \cdots x_{i_{l}}\right\rangle$ where $0 \leqslant i_{0}<\cdots<i_{l} \leqslant k,-1 \leqslant l \leqslant k$, is called an $l$-face of the $k$-simplex $\left\langle x_{0}, \ldots, x_{k}\right\rangle$. The vertices of a $k$-simplex are its 0 -faces.

A $k$-complex is a finite family $\mathbb{Q}=\left\{A_{1}, \ldots, A_{r}\right\}$ of $k$-simplexes such that $A_{i} \cap A_{j}$ is a common face of $A_{i}$ and $A_{j}$ for each $i, j=1, \ldots, r$. The body of $\mathscr{Q}$ is the set $|\mathbb{Q}|=\cup_{i=1}^{n} A_{i}$. The collection $\mathbb{Q}^{\prime},-1 \leqslant l \leqslant k$, of all $l$-faces of $A_{1}, \ldots, A_{r}$ is an $l$-complex called the $l$-skeleton of $\mathfrak{Q}$. 
A subdivision of a $k$-complex $Q=\left\{A_{1}, \ldots, A_{r}\right\}$ is a $k$-complex $\mathscr{B}=\left\{B_{1}, \ldots, B_{s}\right\}$ such that $|\mathscr{Q}|=|\mathscr{B}|$ and each $A_{i}$ is a union of some $B_{j}$ 's. A subdivision $\mathscr{B}$ of $\mathscr{Q}$ is called proper if for each $E, F \in \mathbb{Q}^{l}, 0 \leqslant l \leqslant m$, and each $B \in \mathscr{B}$ we have

$$
(B \cap E \neq \varnothing \text { and } B \cap F \neq \varnothing) \Rightarrow B \cap E \cap F \neq \varnothing .
$$

Since the barycentric subdivision-see Spanier (1966, Chapter 3, Section 3, p. 123) - is proper, we see that each subdivision of $\mathscr{Q}$ can be subdivided further to a proper subdivision of $Q$.

If $A=\left\langle x_{0}, \ldots, x_{k}\right\rangle$ is a $k$-simplex and $x \in A$, denote by st $(x, A)$ the family of all $k$-simplexes among the sets $\left\langle x_{0} \cdots x_{i-1} x x_{i+1} \cdots x_{k}\right\rangle, i=0, \ldots, k$. Clearly, st $(x, A)$ is a subdivision of $\{A\}$ which is proper if and only if $k=1$ and $x$ is not a vertex of $A$.

Throughout, the words "simplex" and "complex" will be used to denote an $m$-simplex and an $m$-complex, respectively. A figure is a set $A \subset \mathbf{R}^{m}$ which is the body of a complex.

The set

$$
\mathbb{Q}=\left\{A_{1}, \ldots, A_{r}, x_{1}, \ldots, x_{r}\right\}
$$

is called a partition if $\left\{A_{1}, \ldots, A_{r}\right\}$ is a complex, the simplexes $A_{1}, \ldots, A_{\mathrm{r}}$ are distinct, and $x_{i}$ is a vertex of $A_{i}, i=1, \ldots, r$. When no confusion is possible we shall denote by $\mathscr{Q}$ both the partition $\left\{A_{1}, \ldots, A_{\mathrm{r}}, x_{1}, \ldots, x_{r}\right\}$ and the complex $\left\{A_{1}, \ldots, A_{\mathrm{r}}\right\}$. If $A$ ia a figure containing $|\mathbb{Q}|$, we call $\mathbb{Q}$ a partition in $A$. If $\delta:|\mathscr{Q}| \rightarrow \mathbf{R}_{+}$, we say that $\mathfrak{Q}$ is $\delta$-fine whenever $d\left(A_{i}\right)<\delta\left(x_{i}\right), i=1, \ldots, r$.

Definition 2.1. Let $\mathbb{Q}$ be a complex. A partition

$$
\mathscr{B}=\left\{B_{1}, \ldots, B_{r} ; x_{1}, \ldots, x_{r}\right\}
$$

is called a partition of $Q$ if $\mathscr{B}$ subdivides $Q$ and

$$
E \subset \operatorname{int}_{|\oplus|} \bigcup\left\{B_{i}: x_{i} \in E\right\}
$$

for each $E \in \mathbb{Q}^{k}, k=0, \ldots, m$.

Clearly, if $\mathscr{B}$ is a partition of $\mathscr{Q}$, then $\mathscr{B}$ is a proper subdivision of $\mathscr{Q}$.

Let $\mathscr{Q}$ be a complex, and let $\delta:|\mathbb{Q}| \rightarrow \mathbf{R}_{+}$be such that

$$
\delta(x) \leqslant \min \left\{|x-y|: y \in\left|\mathbb{Q}^{0}\right|-(x)\right\}
$$

for each $x \in\left|Q^{0}\right|$, and

$$
\delta(x) \leqslant \inf \left\{|x-y|: y \in\left|\mathbb{Q}^{k-1}\right|\right\}
$$

for each $x \in\left|\mathbb{Q}^{k}\right|-\left|\mathbb{Q}^{k-1}\right|, k=1, \ldots, m$. Then it is easy to see that a $\delta$-fine partition $\mathscr{B}$ in $|Q|$ is a partition of $Q$ if and only if $|\mathscr{B}|=|\mathbb{Q}|$. 


\section{Main result}

We begin with some observations about a positive function on a compact set. Let $Q \subset \mathbf{R}^{m}$ be a compact set; $f: Q \rightarrow \mathbf{R}_{+} \cup\{+\infty\}$, and let

$$
\begin{aligned}
& f^{\prime}(x)=\lim _{\varepsilon \rightarrow 0+} \sup \{f(y): y \in Q,|x-y|<\varepsilon\}, \\
& f_{\sim}(x)=\lim _{\varepsilon \rightarrow 0+} \inf \{f(y): y \in Q,|x-y|<\varepsilon\}
\end{aligned}
$$

for each $x \in Q$. Clearly $f_{\sim} \leqslant f^{\sim}$, and it is easy to see that $f_{\sim}$ and $f^{\sim}$ are, respectively, lower and upper semicontinuous.

Lemma 3.1. Let $g=f^{\sim}$, and let $A \subset \mathbf{R}^{m}$ be closed. If $g_{\sim}(x)>0$ for each $x \in A \cap Q$, then there is an $\varepsilon>0$ and a dense subset $D$ of int ${ }_{Q}(A \cap Q)$ such that $f(x)>\varepsilon$ for each $x \in D$.

Proof. Since $A \cap Q$ is compact and $g_{\sim}$ is lower semi-continuous, there is an $\varepsilon>0$ such that

$$
f^{\sim}(x)=g(x) \geqslant g_{\sim}(x)>\varepsilon
$$

for all $x \in A \cap Q$. Thus to each $x \in A \cap Q$ there is a sequence $\left\{x_{n}\right\}$ in $Q$ with $x_{n} \rightarrow x$ and $f\left(x_{n}\right)>\varepsilon, n=1,2, \ldots$ As int ${ }_{Q}(A \cap Q)$ is open in $Q$ is suffices to let

$$
D=\left\{x_{n}: x \in Q, n=1,2, \ldots\right\} \cap \operatorname{int}_{Q}(A \cap Q) .
$$

LEMMA 3.2. Let $g=f^{\sim}$ and $Q^{*}=\left\{x \in Q: g_{\sim}(x)=0\right\}$. Then $Q^{*}$ is compact and nowhere dense in $Q$.

Proof. Since $Q$ is compact and $g_{\sim} \geqslant 0$ is lower semi-continuous, $Q^{*}$ is compact. Suppose there is an $x \in$ int $_{Q} Q^{*}$. Then

$$
U=\{y \in Q:|x-y|<\varepsilon\} \subset Q^{*}
$$

for some $\varepsilon>0$. Using the Baire category theorem in the locally compact space $U$ - see Dugundji (1966, Chapter XI, Theorem 10.3, page 250)-we can find a $c>0$ such that

$$
V=\operatorname{int}_{U}\left[\{z \in U: f(z) \geqslant c\}^{-} \cap U\right]=\text { int }_{Q}\left[\{z \in U: f(z) \geqslant c\}^{-} \cap U\right]
$$

is nonempty. As $g(y) \geqslant c$ for each $y \in\{z \in U: f(z) \geqslant c\}^{-}$, we have $g(y) \geqslant c$ for each $y \in V$. This implies that $g_{\sim}(y) \geqslant c$ for each $y \in V$; for $V$ is open in $Q$. Since $\varnothing \neq V \subset Q^{*}$, we have obtained a contradiction.

THEOREm 3.3. Let $\mathfrak{Q}$ be a complex, and let $\delta:|\mathfrak{Q}| \rightarrow \mathbf{R}_{+}$. Then there is a $\delta$-fine partition of $\mathbb{Q}$. 
Proof. Let $0 \leqslant k \leqslant m, A=|\mathscr{Q}|$, and let $\mathscr{B}$ be a complex with $|\mathscr{B}| \subset A$. Denote by $\Phi_{k}(\mathscr{B})$ the collection of all subdivisions $\left\{C_{1}, \ldots, C_{s}\right\}$ of $\mathscr{B}$ such that $\left\{C_{1}, \ldots, C_{q} ; y_{1}, \ldots, y_{q}\right\}, 1 \leqslant q \leqslant s$, is a $\delta$-fine partition in $|\Re|$ with $\left\{y_{1}, \ldots, y_{q}\right\} \subset$ $\left|93^{k}\right|$, and

$$
E \subset \operatorname{int}_{\left|\mathbb{P}_{\mid}\right|} \cup\left\{C_{j}: y_{j} \in E\right\}
$$

for each $E \in \mathscr{B}^{l}, l=0, \ldots, k$. We shall prove by induction that $\Phi_{k}(\mathscr{B}) \neq \varnothing$. This is true if $k=0$; for $\Phi_{0}(\mathscr{B})$ contains every proper subdivision $\mathcal{C}$ of $\mathscr{B}$ with

$$
\operatorname{mesh} C<\min \left\{\delta(x): x \in \mathscr{B}^{0}\right\} .
$$

Assume it is true for $k-1$ with $1 \leqslant k \leqslant m$. Clearly, it suffices to show that $\Phi_{k}(\mathbb{Q}) \neq \varnothing$.

Claim (i). Let $\mathscr{B}$ be a complex with $|\mathscr{B}| \subset A$, and let $\mathscr{B}$ ' be a subdivision of $\mathscr{B}$. Then $\Phi_{k-1}\left(\mathscr{B}^{\prime}\right) \subset \Phi_{k-1}\left(\mathscr{B}^{\prime}\right)$. In particular, given $\varepsilon>0, \Phi_{k-1}(\mathscr{B})$ contains a complex $\mathcal{C}$ with mesh $\mathcal{C}<\varepsilon$.

Proof. Choose a complex $e=\left\{C_{1}, \ldots, C_{s}\right\}$ in $\Phi_{k-1}\left(\mathscr{G}^{\prime}\right)$, and let $\left\{C_{1}, \ldots, C_{q} ; y_{1}, \ldots, y_{q}\right\}, 1 \leqslant q \leqslant s$, be the appropriate $\delta$-fine partition in $\left|\mathscr{B}^{\prime}\right|=$ $|\mathscr{B}|$. After a suitable reordering we may assume that

$$
\left\{y_{1}, \ldots, y_{p}\right\}=\left\{y_{1}, \ldots, y_{q}\right\} \cap\left|\mathscr{B}^{k-1}\right|,
$$

$1 \leqslant p \leqslant q$. Considering the partition $\left\{C_{1}, \ldots, C_{p} ; y_{1}, \ldots, y_{p}\right\}$, it is easy to see that $\mathcal{C} \in \Phi_{k-1}(\mathscr{B})$; for $\mathscr{B}^{\prime \prime} \cap \mathscr{B}^{l}$ subdivides $\mathscr{B}^{l}, l=0, \ldots, m$. Now the rest of the claim follows from Spanier (1966, Chapter 3, Section 3, Theorem 14, page 125).

Claim (ii). Let $\mathscr{B}$ be a complex with $B=|\mathscr{B}| \subset A$, let $Q \subset\left|\mathscr{B}^{k}\right|$, and let $D$ be a dense subset of $Q-\left|B^{k-1}\right|$. Suppose that $\delta(x)>\varepsilon$ for some $\varepsilon>0$ and each $x \in D$. Then there is a proper subdivision $\left\{C_{1}, \ldots, C_{s}\right\}$ of $\mathscr{B}$ such that $\left\{C_{1}, \ldots, C_{q} ; y_{1}, \ldots, y_{q}\right\}, 1 \leqslant q \leqslant s$, is a $\delta$-fine partition in $B$ with $\left\{y_{1}, \ldots, y_{q}\right\} \subset$ $\left|\mathscr{B}^{k}\right|$,

$$
E \subset \operatorname{int}_{B} \bigcup\left\{C_{j}: y_{j} \in E\right\}
$$

for each $E \in \mathscr{B}^{\prime}, l=0, \ldots, k-1$, and

$$
\left\{y_{1}, \ldots, y_{q}\right\} \cup Q \subset \operatorname{int}_{B} \bigcup_{j=1}^{q} C_{j} \text {. }
$$

Proof. By Claim (i), there is a proper subdivision $\mathcal{C}=\left\{C_{1}, \ldots, C_{s}\right\}$ of $\mathscr{B}$ with mesh $e<\varepsilon$ and such that $\left\{C_{1}, \ldots, C_{p} ; y_{1}, \ldots, y_{p}\right\}, 1 \leqslant p \leqslant s$, is a $\delta$-fine partition 
in $B$ with $\left\{y_{1}, \ldots, y_{p}\right\} \subset\left|\mathscr{B}^{k-1}\right|$, and

$$
E \subset \operatorname{int}_{B} \bigcup\left\{C_{j}: y_{j} \in E\right\}
$$

for each $E \in \mathscr{B}^{l}, l=0, \ldots, k-1$. In particular, $C_{j} \cap\left|\mathscr{B}^{k-1}\right|=\varnothing$ for $j=$ $p+1, \ldots, s$. After a suitable reordering we may assume that $C_{j} \cap Q \neq \varnothing$ for $j=$ $p+1, \ldots, q$, and $C_{j} \cap Q=\varnothing$ for $j=q+1, \ldots, s ; p \leqslant q \leqslant s$. Thus

$$
Q \subset \operatorname{int}_{B} \bigcup_{j=1}^{q} C_{j} \text {. }
$$

If $C_{j}, p<j \leqslant q$, has no vertex in $Q$, find an $x \in C_{j} \cap Q$ and replace each $C_{i}$, $p<i \leqslant q$, containing $x$ by st $\left(x, C_{i}\right)$. By repeating this process finitely many times, we obtain a subdivision of $\left\{C_{p+1}, \ldots, C_{q}\right\}$ such that each simplex of this subdivision has a vertex in $Q$. Thus with no loss of generality we may assume that each $C_{j}, j=p+1, \ldots, q$, already has a vertex in $Q$. Let $\left\{z_{1}, \ldots, z_{n}\right\}$ be all vertices of $C_{1}, \ldots, C_{q}$. If $z_{i} \in Q$ and $z_{i}$ is a vertex of $C_{j}$ with $p<j \leqslant q$, choose a $z_{i}^{\prime} \in D$; otherwise let $z_{i}^{\prime}=z_{i} ; i=1, \ldots, n$. If $C_{j}=\left\langle z_{i_{0}} \cdots z_{i_{m}}\right\rangle$, set $C_{j}^{\prime}=\left\{z_{i_{0}}^{\prime} \cdots z_{i_{m}}^{\prime}\right\}$, $j=1, \ldots, q$. Since each $z_{i}$ with $z_{i}^{\prime} \neq z_{i}, 1 \leqslant i \leqslant n$, lies in

$$
\left(\text { int }_{B} \bigcup_{j=1}^{q} C_{j}\right) \cap\left(Q-\left|\mathscr{B}^{k-1}\right|\right),
$$

and since $D$ is dense in $Q-\left|\mathscr{B}^{k-1}\right|$, we can choose $z_{i}^{\prime}$ in $\left|\mathscr{B}^{k}\right|-\left|\mathscr{B}^{k-1}\right|$ and so close to $z_{i}$ that $e^{\prime}=\left\{C_{1}^{\prime}, \ldots, C_{q}^{\prime}, C_{q+1}, \ldots, C_{s}\right\}$ is a proper subdivision of $\mathscr{B}$ with mesh $e^{\prime}<\varepsilon$. Moreover, each $C_{j}^{\prime}, p<j \leqslant q$, has a vertex $y_{j}^{\prime} \in D$. It follows that

$$
\left\{C_{1}^{\prime}, \ldots, C_{q}^{\prime} ; y_{1}, \ldots, y_{p}, y_{p+1}^{\prime}, \ldots, y_{q}^{\prime}\right\}
$$

is the desired $\delta$-fine partition in $B$, and the claim is proved.

Let $Q_{0}=\left|Q^{k}\right|$, and suppose that a compact set $Q_{\alpha} \subset\left|\mathbb{Q}^{k}\right|$ has been defined for each ordinal $\alpha<\beta$. If $\beta$ is a limit ordinal, set $Q_{\beta}=\bigcap_{\alpha<\beta} Q_{\alpha}$. If $\beta=\alpha+1$, let $\delta_{\alpha}$ be the restriction of $\delta$ to $Q_{\alpha}, \Delta^{\alpha}=\delta_{\alpha}^{\sim}$, and set

$$
Q_{\beta}=\left\{x \in Q_{\alpha}: \Delta_{\sim}^{\alpha}(x)=0\right\} \text {. }
$$

By Lemma 3.2, $Q_{\alpha+1} \subsetneq Q_{\alpha}$ whenever $Q_{\alpha} \neq \varnothing$. Thus there is the first ordinal $\kappa$ with $Q_{\kappa}=\varnothing$. From our construction it is clear that $\kappa=\gamma+1$. It follows from Kuratowski (1966, Section 24, II, Theorem 2) that $\kappa$ is a countable ordinal but we shall not need this.

Let $\Gamma$ be the set of all ordinals $\alpha \leqslant \gamma$ for which there is a proper subdivision $\mathscr{G}_{B}=\left\{B_{1}, \ldots, B_{r}\right\}$ of $\Theta$ such that $\left\{B_{1}, \ldots, B_{p} ; x_{1}, \ldots, x_{p}\right\}, 1 \leqslant p \leqslant r$, is a $\delta$-fine partition in $A$ with $\left\{x_{1}, \ldots, x_{p}\right\} \subset\left|\mathbb{Q}^{k}\right|$,

$$
E \subset \operatorname{int}_{A} \bigcup\left\{B_{i}: x_{i} \in E\right\}
$$


for each $E \in \Re^{l}, l=0, \ldots, k-1$, and

$$
\left\{x_{1}, \ldots, x_{p}\right\} \cup Q_{\alpha} \subset \operatorname{int}_{A} \bigcup_{i=1}^{p} B_{i} .
$$

As the subdivision $\mathscr{B}$ is proper our induction will be completed by showing that $0 \in \Gamma$.

It follows immediately from Lemma 3.1 and Claim (ii) that $\gamma \in \Gamma$.

Claim (iii). If $\beta \in \Gamma$ is a limit ordinal, then there is an $\alpha \in \Gamma$ with $\alpha<\beta$.

Proof. If $\left\{B_{1}, \ldots, B_{p} ; x_{1}, \ldots, x_{p}\right\}$ is a $\delta$-fine partition in $A$ associated with $\beta \in \Gamma$, then

$$
Q_{\beta} \subset G=\operatorname{int}_{A} \bigcup_{i=1}^{p} B_{i} .
$$

Since $\left\{Q_{\alpha}-G: \alpha<\beta\right\}$ is a chain of compact sets and

$$
\bigcap_{\alpha<\beta}\left(Q_{\alpha}-G\right)=Q_{\beta}-G=\varnothing,
$$

we have $Q_{\alpha} \subset G$ for some $\alpha<\beta$. It follows that $\alpha \in \Gamma$.

Claim (iv). If $\alpha+1 \in \Gamma$, then $\alpha \in \Gamma$.

Proof. There is a proper subdivision $\mathscr{B}=\left\{B_{1}, \ldots, B_{r}\right\}$ of $\mathbb{Q}$ such that $\left\{B_{1}, \ldots, B_{p} ; x_{1}, \ldots, x_{p}\right\}, 1 \leqslant p \leqslant r$, is a $\delta$-fine partition in $A$ with $\left\{x_{1}, \ldots, x_{p}\right\} \subset$ $\left|Q^{k}\right|$,

$$
E \subset \operatorname{int}_{A} \cup\left\{B_{i}: x_{i} \in E\right\}
$$

for each $E \in \mathscr{B}^{l}, l=0, \ldots, k-1$, and

$$
\left\{x_{1}, \ldots, x_{p}\right\} \cup Q_{\alpha+1} \subset \operatorname{int}_{A} \bigcup_{i=1}^{p} B_{i} .
$$

Let $\mathscr{Q}=\left\{B_{p+1}, \ldots, B_{r}\right\}$. By Lemma 3.1 there is an $\varepsilon>0$ and a dense subset $D$ of $|\mathscr{D}| \cap Q_{\alpha}-\left|\mathcal{D}^{k-1}\right|$ such that $\delta(x)>\varepsilon$ for each $x \in D$; for $\Delta_{\sim}^{\alpha}(x)>0$ for every $x \in \mid$ OD $\mid \cap Q_{\alpha}$, and

$$
|\mathscr{D}| \cap Q_{\alpha}-\left|\mathscr{D}^{k-1}\right| \subset \text { int }_{Q_{\alpha}}\left(|\mathscr{D}| \cap Q_{\alpha}\right)
$$

Using Claim (ii), we can find a proper subdivision $\left\{C_{1}, \ldots, C_{s}\right\}$ of $\mathscr{D}$ such that $\left\{C_{1}, \ldots, C_{q} ; y_{1}, \ldots, y_{q}\right\}, 1 \leqslant q \leqslant s$, is a $\delta$-fine partition in $|\mathscr{D}|$ with $\left\{y_{1}, \ldots, y_{q}\right\} \subset$ $\left|\mathcal{Q}^{k}\right|$

$$
E \subset \operatorname{int}_{|\mathscr{D}|} \cup\left\{C_{j}: y_{j} \in E\right\}
$$


for each $E \in \mathscr{Q}^{l}, l=0, \ldots, k-1$, and

$$
\left\{y_{1}, \ldots, y_{q}\right\} \cup\left(|\mathscr{Q}| \cap Q_{\alpha}\right) \subset \operatorname{int}_{|\mathscr{Q}|} \bigcup_{j=1}^{q} C_{j} .
$$

Since $\left\{x_{1}, \ldots, x_{p}\right\} \subset$ int $_{A} \cup_{i=1}^{p} B_{i}$, there is a subdivision $\left\{P_{1}, \ldots, P_{n}\right\}$ of $\left\{B_{1}, \ldots, B_{p}\right\}$ such that each $P_{j}, 1 \leqslant j \leqslant n$, has a vertex $z_{j}$ in $\left\{x_{1}, \ldots, x_{p}\right\}$ and $\left\{P_{1}, \ldots, P_{n}, C_{1}, \ldots, C_{s}\right\}$ is a subdivision of $\mathscr{G}$. After a suitable reordering we may assume that

$$
\left\{y_{1}, \ldots, y_{t}\right\}=\left\{y_{1}, \ldots, y_{q}\right\} \cap\left|\mathbb{Q}^{k}\right|,
$$

$1 \leqslant t \leqslant q$. Considering the partition $\left\{P_{1}, \ldots, P_{n}, C_{1}, \ldots, C_{t} ; z_{1}, \ldots, z_{n}, y_{1}, \ldots, y_{t}\right\}$ it is easy to see that $\alpha \in \Gamma$; for $\mathbb{B}^{l} \cap \mathbb{Q}^{l}$ subdivides $\mathbb{Q}^{l}, l=0, \ldots, m$.

As every decreasing sequence of ordinals is finite-see Dugundji (1966, Chapter II, Theorem 6.4 (5), page 43) - it follows from Claims (iii) and (iv) that $0 \in \Gamma$, and the theorem is proved.

The next proposition is the main motivation for Definition 2.1. It shows that a $\delta$-fine partition of a subcomplex can be always extended to a $\delta$-fine partition of the whole complex.

Proposition 3.4. Let $\mathscr{Q}$ be a complex, $\delta:|\Theta| \rightarrow \mathbf{R}_{+}$, and let $\mathscr{G}$ be a subcomplex of $Q$. If $\mathscr{B}^{\prime}$ is a $\delta$-fine partition of $\mathscr{B}$, then there is a $\delta$-fine partition $\mathcal{C}^{\prime}$ of $\mathbb{C}=\mathbb{Q}-\mathscr{B}$ such that $\mathscr{B}^{\prime} \cup \mathcal{C}^{\prime}$ is a $\delta$-fine partition of $Q$.

Proof. Without loss of generality, we may assume that $\mathcal{C}$ consists of a single $m$-simplex $C$. Let $\mathscr{B}^{\prime}=\left\{B_{1}, \ldots, B_{p} ; x_{1}, \ldots, x_{p}\right\}$, and for $k=0, \ldots, m-1$, let $\mathscr{P}_{k}=\left\{C_{1}, \ldots, C_{q} ; y_{1}, \ldots, y_{q}\right\}$ be a $\delta$-fine partition in $C$ with the following properties:

(i) $\mathscr{P}_{k} \cup \mathscr{S B}^{\prime}$ is a complex, and $\left\{y_{1}, \ldots, y_{q}\right\} \subset\left\{x_{1}, \ldots, x_{p}\right\}$;

(ii) $E \subset$ int $_{C} \cup\left\{C_{j}: y_{j} \in E\right\}$ for each $E \in \mathbb{C}^{l} \cap \mathfrak{B}^{\prime}, l=0, \ldots, k$;

(iii) $\mathscr{P}_{k}$ can be extended to a proper subdivision of $\mathrm{e}$;

(iv) the $(m-1)$-dimensional face $E_{j}$ of $C_{j}$ opposite to $y_{j}, 1 \leqslant j \leqslant q$, is perpendicular to each $E \in \mathcal{C}^{\prime}, 1 \leqslant l<m$, for which $E \cap E_{j} \neq \varnothing$.

It is easy to see that $\mathscr{P}_{0}$ exists. Assuming the existence of $\mathscr{P}_{k}, 0 \leqslant k \leqslant m-2$, we shall prove that $\mathscr{P}_{k+1}$ exists.

Given $E \in \mathcal{C}^{k+1} \cap \mathscr{B}^{k+1}$, choose a $B \in \mathscr{B}$ with $E \subset B$. After a suitable reordering, we may assume that $B_{1}, \ldots, B_{t}, 0 \leqslant t<p$, are all elements from $\mathscr{B}^{\prime}$ for which the associated vertices $x_{1}, \ldots, x_{t}$ belong to $E-\left|B^{k}\right|$. Using (i) and (iv), we can construct a $\delta$-fine partition $\mathscr{P}_{E}=\left\{D_{1}, \ldots, D_{n} ; z_{1}, \ldots, z_{n}\right\}$ in $C$ such that

(a) $\mathscr{P}_{E} \cup \mathscr{P}_{k} \cup \mathscr{B}^{\prime}$ is a complex, and $\left\{z_{1}, \ldots, z_{n}\right\}=\left\{x_{1}, \ldots, x_{t}\right\}$; 
(b) $E \subset$ int $_{c}\left[\left(\cup\left\{C_{j} \in \mathscr{P}_{k} ; y_{j} \in E\right\}\right) \cup\left(\cup_{i=1}^{n} D_{i}\right)\right]$;

(c) $\mathscr{P}_{E}$ can be extended into a proper subdivision of $\mathrm{C}$;

(d) the $(m-1)$-dimensional face $F_{i}$ of $D_{i}$ opposite to $z_{i}, 1 \leqslant i \leqslant n$, is perpendicular to each $F \in \mathcal{C}^{\prime}, 1 \leqslant l<m$, for which $F \cap F_{i} \neq \varnothing$.

Roughly speaking, $\mathscr{P}_{E}$ is obtained by an appropriate squashing and refining of the partition $\left\{B_{1}, \ldots, B_{t} ; x_{1}, \ldots, x_{t}\right\}$ flipped across $E$ into $C$. Now it follows from (ii) that

$$
\mathscr{P}_{k+1}=\mathscr{P}_{k} \cup\left[\cup\left\{\mathscr{P}_{E}: E \in \mathcal{C}^{k+1} \cap \mathscr{B}^{k+1}\right\}\right] .
$$

$\mathscr{P}_{m-1}=\left\{P_{1}, \ldots, P_{r} ; u_{1}, \ldots, u_{r}\right\}$. By (iii), there is a complex $\mathscr{Q}$ such that $\mathscr{Q} \cup$ $\mathscr{P}_{m-1}$ is a proper subdivision of $\mathcal{C}$. Applying Theorem 3.3, we can find a $\delta$-fine partition $\left\{S_{1}, \ldots, S_{s} ; v_{1}, \ldots, v_{s}\right\}$ of $\mathscr{Q}$. Since

$$
\left\{u_{1}, \ldots, u_{r}\right\} \subset \text { int }_{C} \bigcup_{i=1}^{r} P_{r},
$$

there is a subdivision $\left\{Q_{1}, \ldots, Q_{l}\right\}$ of $\mathscr{P}_{m-1}$ such that each $Q_{j}, 1 \leqslant j \leqslant l$, has a vertex $w_{j}$ in $\left\{u_{1}, \ldots, u_{r}\right\}$, and $\left\{Q_{1}, \ldots, Q_{l}, S_{1}, \ldots, S_{s}\right\}$ subdivides $\mathcal{C}$. The proof is completed by letting

$$
e^{\prime}=\left\{Q_{1}, \ldots, Q_{l}, S_{1}, \ldots, S_{s} ; w_{1}, \ldots, w_{l}, v_{1}, \ldots, v_{s}\right\} .
$$

Let $\mathscr{Q}$ be a complex, and $\delta:|\mathfrak{Q}| \rightarrow \mathbf{R}_{+}$. It is natural to investigate whether there is a $\delta$-fine partition $\left\{A_{1}, \ldots, A_{n} ; x_{1}, \ldots, x_{n}\right\}$ of $\mathscr{Q}$ whose simplexes satisfy some regularity condition - see Whitney (1957, Chapter IV, Section 4). In particular, one would like to know whether the solid angle of $A_{i}$ at $x_{i}, i=1, \ldots, n$, is bounded away from zero uniformly with respect to $\delta$. The following example shows that this cannot be achieved.

ExAmple 3.5. Set $m=2$, and let $A$ be a 2 -simplex in $\mathbf{R}^{2}$ such that $\left\{x \in R^{2}\right.$ : $|x|<1\} \subset A$. Denote by 0 the origin in $\mathbf{R}^{2}$, and choose $0<\varepsilon<\frac{1}{2}$. For $x \in X$, set

$$
\delta_{\varepsilon}(x)= \begin{cases}\varepsilon|x| & \text { if } x \neq 0, \\ \varepsilon & \text { if } x=0,\end{cases}
$$

and let $\left\{A_{1}, \ldots, A_{n} ; x_{1}, \ldots, x_{n}\right\}$ be a $\delta$-fine partition of $\{A\}$. By the choice of $\delta_{\varepsilon}$, $n \geqslant 2$ and after a suitable reordering, $A_{1}=\langle 0 x y\rangle, x_{1}=0$, and $A_{2}=\langle x y z\rangle$ for some $x, y, z$ in $A$. With no loss of generality, we may assume that $x_{2}=x$ or $x_{2}=z$. If $x_{2}=z$, then $|x-y|<\varepsilon|z|$ and $|z-x|<\varepsilon|z|$. As $|z| \leqslant|x|+\mid z-$ $x \mid$, we have $|z|<|x|+\varepsilon|z|$, and consequently

$$
|x-y|<\varepsilon|z|<\frac{\varepsilon}{1-\varepsilon}|x|<2 \varepsilon|x| \text {. }
$$

However, if $x_{2}=x$, then again

$$
|x-y|<\varepsilon|x|<2 \varepsilon|x| \text {. }
$$


From $|x|-|x-y| \leqslant|y| \leqslant|x|+|x-y|$ we obtain

$$
1-2 \varepsilon<1-\frac{|x-y|}{|x|} \leqslant \frac{|y|}{|x|} \leqslant 1+\frac{|x-y|}{|x|}<1+2 \varepsilon .
$$

If $\alpha$ is the angle of $A_{1}$ at 0 , then

$$
\begin{aligned}
\cos \alpha & =\frac{1}{2|x||y|}\left(|x|^{2}+|y|^{2}-|x-y|^{2}\right) \\
& =\frac{1}{2}\left(\frac{|x|}{|y|}+\frac{|y|}{|x|}-\frac{|x-y|^{2}}{|x|^{2}} \cdot \frac{|x|}{|y|}\right) \geqslant 1-2 \varepsilon .
\end{aligned}
$$

It follows that $\alpha \rightarrow 0$ as $\varepsilon \rightarrow 0$.

\section{An application}

For $x \in \mathbf{R}^{m}$ and a sequence $\left\{B_{n}\right\}$ of simplexes, we write $B_{n} \rightarrow x$ whenever $x$ is a common vertex of each $B_{n}, n=1,2, \ldots$, and $d\left(B_{n}\right) \rightarrow 0$. If $A$ is a simplex, denote by $\sigma_{A}$ the family of all simplexes contained in $A$. Finally, let $\lambda$ be the $m$-dimensional Lebesgue measure in $\mathbf{R}^{m}$.

Let $A$ be a simplex, $x \in A$, and let $\phi: \sigma_{A} \rightarrow \mathbf{R}$. The extended real number

$$
{ }_{*} \phi(x)=\inf \left[\liminf \frac{\phi\left(B_{n}\right)}{\lambda\left(B_{n}\right)}\right]
$$

where the infimum is taken over all sequences $\left\{B_{n}\right\}$ from $\sigma_{A}$ with $B_{n} \rightarrow x$, is called the lower derivate of $\phi$ at $x$.

Definition 4.1. Let $A$ be a simplex. A function $\phi: \sigma_{A} \rightarrow \mathbf{R}$ is called superadditive if

$$
\phi(A) \geqslant \Sigma\{\phi(B): B \in \mathscr{B}\}
$$

for each complex $\mathscr{B}$ with $|\mathscr{B}|=A$.

We note that Definition 4.1 is substantially weaker than the usual definition of superadditivity.

TheOREM 4.2. Let $A$ be a simplex, and let $\phi: \sigma_{A} \rightarrow \mathbf{R}$ be a superadditive function. If ${ }_{*} \phi(x) \geqslant 0$ for each $x \in A$, then $\phi(A) \geqslant 0$.

Proof. Choose an $\varepsilon>0$, and let $\psi=\phi+\varepsilon \lambda$. Then $\psi: \sigma_{A} \rightarrow \mathbf{R}$ is superadditive, and

$$
{ }_{*} \psi(x) \geqslant{ }_{*} \phi(x)+\varepsilon>0
$$


for each $x \in A$. Thus given $x \in A$, there is a $\delta(x)>0$ such that $\psi(B)>0$ for each $B \in \sigma_{A}$ with a vertex $x$ and $d(B)<\delta(x)$. By Theorem 3.3, we can find a $\delta$-fine partition $\left\{B_{1}, \ldots, B_{p} ; x_{1}, \ldots, x_{p}\right\}$ of $\{A\}$. Since

$$
\phi(A)+\varepsilon \lambda(A)=\psi(A) \geqslant \sum_{i=1}^{p} \psi\left(B_{i}\right)>0,
$$

the theorem follows from the arbitrariness of $\varepsilon$.

Theorem 4.2 is actually equivalent to the following weaker version of Theorem 3.3:

Given a simplex $A$ and a $\delta: A \rightarrow \mathbf{R}_{+}$, there is a $\delta$-fine partition $\mathbb{Q}=$ $\left\{A_{1}, \ldots, A_{n} ; x_{1}, \ldots, x_{n}\right\}$ in $A$ with $|\mathscr{Q}|=A$.

To see this, suppose that there is a $\delta: A \rightarrow \mathbf{R}_{+}$such that no such $\delta$-fine partition $\mathbb{Q}$ in $A$ exists. For $B \in \sigma_{A}$, let $\phi(B)=0$ if $B$ has a vertex $x$ with $d(B)<\delta(x)$, and $\phi(B)=-1$ otherwise. Then $\phi: \sigma_{A} \rightarrow \mathbf{R}$ is superadditive, $\phi(A)$ $=-1$, and ${ }_{*} \phi(x)=0$ for each $x \in A$.

This paper was prepared while the author visited the University of South Africa in Pretoria, the Royal Institute of Technology in Stockholm and the Monash University in Melbourne.

\section{References}

J. Dugundji (1966), Topology (Allyn and Bacon, Boston).

R. Henstock (1968), 'A Riemann-type integral of Lebesgue power', Canad. J. Math. 20, 79-87.

K. Kuratowski (1966), Topology (Academic Press, New York).

J. Kurzweil (1957), 'Generalized ordinary differential equations and continuous dependence on a parameter,' Czechoslovak Math. J. 82, 418-446.

E. Spanier (1966), Algebraic topology (McGraw-Hill, New York).

H. Whitney (1957), Geometric integration theory (Princeton Univ. Press, Princeton).

Department of Mathematics

University of California

Davis, California 95616

U.S.A. 